\title{
Exotic matter in neutron stars and the equation of state
}

\section{S. Schramm*}

Frankfurt Institute for Advanced Studies, D-60438 Frankfurt, Germany

E-mail: schrammefias.uni-frankfurt.de

\section{Dexheimer}

Department of Physics, Kent State University, Kent OH, USA

E-mail: volexheimakent.edu

\section{J. Steinheimer}

Frankfurt Institute for Advanced Studies, D-60438 Frankfurt, Germany

E-mail: steinheimerefias.uni-frankfurt.de

\section{A. Mukherjee}

Frankfurt Institute for Advanced Studies, D-60438 Frankfurt, Germany

E-mail: mukherjee@fias.uni-frankfurt.de

\begin{abstract}
The interior of neutron stars contains strongly interacting matter at extremely high densities, perhaps up to ten times nuclear matter saturation density. Under such conditions, it is natural to assume that exotic matter beyond nucleons appears. This includes hyperons and other baryonic resonances, meson condensates, as well as free quarks. In this article, we discuss some implications for such matter on neutron star properties.
\end{abstract}

Frontier Research in Astrophysics - III (FRAPWS2018)

28 May - 2 June 2018

Mondello (Palermo), Italy

${ }^{*}$ Speaker. 


\section{Introduction}

In recent years many new observations of neutron star properties were obtained and, in the time ahead, a whole array of upcoming and planned missions and detectors will add to our knowledge of this exciting field of physics. From the perspective of strong interaction physics the interior of neutron stars or , more generally, compact stars, is in particular of great interest, as it constitutes the densest low-temperature environment to be found in nature. This is in contrast to the fireball created in high-energy heavy-ion collisions, where extreme temperatures beyond $200 \mathrm{MeV}$ can be reached. Here, depending on the beam energy, net densities (particle minus anti-particle densities) can also reach ten times nuclear matter density or more, albeit at high temperature. This region of conditions will be the center of interest for the upcoming facilities FAIR at GSI and NICA in Dubna. Very intriguingly, proto-neutron stars and, in particular, neutron star mergers, as first observed in 2017 [1], can reach high temperatures (in the latter case perhaps up to $100 \mathrm{MeV}$ ). Thus, strongly interacting matter is created in stars at conditions similar to the fireball of heavy-ion collisions at around $1 \mathrm{GeV}$ per nucleon beam energy, though at different isospin of the matter. Still, compact star and heavy-ion physics start to overlap, which demands theoretical formulations that can describe both types of systems. Such an approach will be discussed in the following.

\section{Model Formalism}

The hadronic part of the model, termed chiral mean field model (CMF), is based on an extension of the chiral $\sigma-\omega$ model to include strangeness in a flavor-SU(3) formulation. Details of this formulation have been discussed in a number of publications $[2,3,4]$. Concentrating on the main properties, baryons interact via their coupling to mesonic mean fields. The coupling is linear, i.e. the Lagrangian contains the coupling term:

$$
\mathscr{L}_{\text {int }}=-\sum_{i} \bar{B}_{i}\left[\gamma_{0}\left(g_{i \omega} \omega+g_{i \rho} \rho+g_{i \phi} \phi\right)+g_{i \sigma} \sigma+g_{i \delta} \delta+g_{i \zeta} \zeta\right] B_{i} .
$$

The sum over the baryons contain nucleons and hyperons, whereas the mesons include the lowest SU(3) multiplets. In particular, for static matter the mean fields of the 0th components of the vector mesons $\omega$ (isoscalar), $\rho$ (isovector), and $\phi$ (strange - antistrange quark state) contribute, together with the corresponding scalar fields, here denoted as $\sigma, \delta$, and $\zeta$. Via their nonlinear self-interactions [2], the scalar fields $\sigma$ and $\zeta$ attain non-zero vacuum expectation values, which generate the baryon masses through Eq. 2.1. In addition, due to the change of the fields in hot and/or dense matter the baryon masses are modified in the medium. While scalar fields in general lead to attractive interactions, the vector mean fields generate repulsion, which becomes the dominant part of the interaction for very dense systems.

As argued before, a model description for stellar and heavy-ion matter should include quarks as degrees of freedom. This is done in the CMFq approach by coupling quark fields to the mean fields that effectively represent quark condensates, by extending the sum in Eq. 2.1 to include quarks [5, 6]. In addition, an effective field with a respective potential is introduced. This field runs from zero to values close to one towards high densities and/or temperatures, describing the deconfinement 
transition analogously to the Polyakov loop field in the so-called PNJL quark models [7, 8]. This field either couples to the quark distribution functions or the quark mass to suppress quarks in the hadronic phase at lower temperatures and densities. Details of this formulation can be found in Refs. [5, 6] .

A further recent extension of the model is the treatment of the baryons in the parity-doublet formulation of chiral symmetry $[9,10,11]$. In this approach the baryons are paired with the negative parity excited baryonic states into doublets, very much like the scalar $\sigma$ meson and pseudoscalar $\pi$ meson is treated as doublet in linear sigma models. The signature of chiral symmetry restoration in this case is the degeneracy of the doublet states. Recent QCD lattice calculations of the temperature of the mass dependence of the doublet states appear to be in accordance with such a picture $[12,13]$. From a practical viewpoint, the main difference in the calculation is that the baryonic quasi-particle energies, e.g. for the case of nucleon, now read:

$$
m_{N}^{*}( \pm)=\sqrt{\left(g_{N \sigma} \sigma+<\tau_{3}>g_{N \delta} \delta\right)^{2}+m_{0}^{2}} \mp g_{N \sigma}^{\prime} \sigma \mp<\tau_{3}>g_{N \delta}^{\prime} \delta
$$

where the isospin factor $\left\langle\tau_{3}\right\rangle$ is +1 for protons and -1 for neutrons, respectively. The \pm sign factor refers to the two opposite parity states. As is obvious from this expression, the masses become degenerate for the case of vanishing fields. The parity-doublet model allows for a second set of coupling parameters $g^{\prime}$ to the scalar fields as shown in Eq. 2.2, which describe the mass splitting of the parity doublet states and can be determined by their vacuum values of the splittings.

\section{Results}

The parity-doublet model as described above has recently been used for calculations of isospin symmetric matter, relevant for heavy-ion collisions [10], as well as for beta-equilibrated neutron star matter, discussed in Ref. [9]. In a slightly adjusted fit to this reference (adjusting the parameter $m_{0 q}$ in Eq. 5 of this paper to $215 \mathrm{MeV}$ ), the resulting neutron star masses as functions of the central energy density are shown in Fig. 1. In agreement with observations of heavy neutron stars [14, 15] and limits for maximum star masses deduced from the gravitational wave signal of the neutron star merger event and its measured afterglow [16, 17], the maximum mass reached is about 2 solar masses with a central density of 7.5 times nuclear matter saturation density. Fig. 2 depicts the normalized quark fraction of the baryon number density as function of baryon number chemical potential $\mu_{B}$. In the center of the heaviest star (in Fig. 1) the value of $\mu_{B}$ reaches $1730 \mathrm{MeV}$. From the figure one can see that there is a phase of mixed matter of baryons and quarks over a wide range of chemical potential, making the compact star solutions within this model truly hybrid stars, consisting of hadrons and quarks. The jump in the density, seen in Fig. 2, arises from a weak firstorder phase transition. Fig. 2 also contains the results for the amount of strangeness per baryon $f_{s}$. Due to the early occurrence of quarks, hyperons are suppressed and strange particles only come in at high densities when the strange quark appears.

In general, hybrid star phases might appear at later stages of the neutron star evolution. This is possible if one looks at rotational as well as effects from strong magnetic fields. As deduced from the observation of magnetars, the stellar surface magnetic fields might reach values of $10^{15}$ Gauss or higher $[18,19,20]$. The magnetic field in the interior of stars could be significantly stronger, still. 


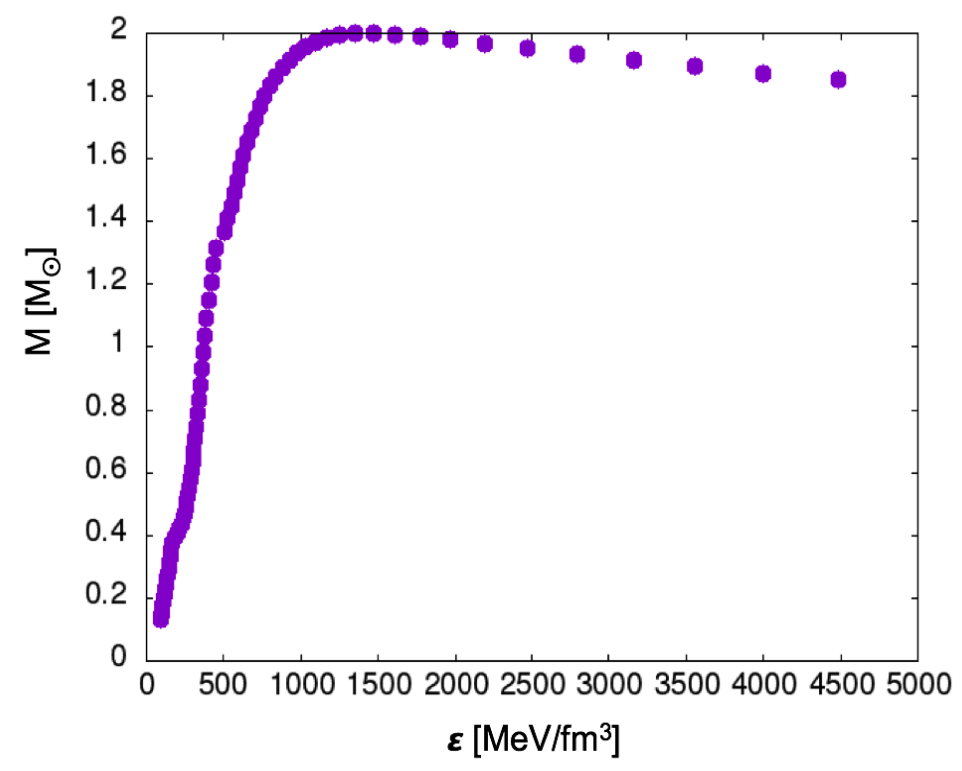

Figure 1: Mass of neutron star as function of central energy density. The structure at about 1.3 solar masses originates from the population onset of the neutron parity partner.

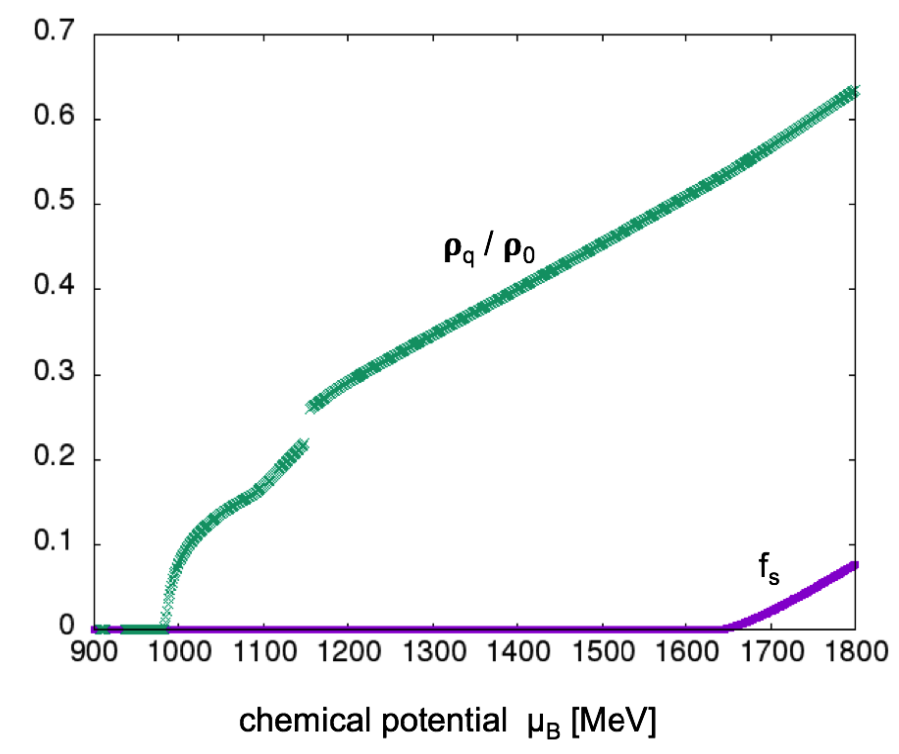

Figure 2: Normalized quark fraction of beta-equilibrated stellar matter as function of baryon chemical potential. The strangeness fraction $f_{s}$ of matter is also shown. 
The effect of such high magnetic fields on a hybrid star has been studied in Refs. [21, 22], where a similar quark-hadron model as described above, but with a stronger phase transition from hadrons to quarks has been employed [5]. In Fig. 3 the composition of a star with fixed baryon number for different magnetic field strengths is shown, starting from the bottom right panel with a central field $B_{c}=9.1 \times 10^{17} \mathrm{G}$ down to vanishing field (top left panel), which corresponds to snapshots in time of a star with decaying fields. The pressure from the magnetic field leads to a strong reduction of the stellar central density. Therefore, for high fields the star consists only of nucleons (plus leptons). With decaying field, hyperons appear and, in the end, quarks are populated in a first-order phase transition in the core of the star generating a hybrid star. Such change of composition has an impact on radius and moment of inertia of the star, leading to potential observable consequences in the spin-down or spin-up of the star as discussed in Ref. [21]. Note that this behavior does not rely on fast rotation of the star and is, therefore, applicable to magnetars, which have so far been observed to have slow rotation. In summary, model calculations of hybrid stars with an extended

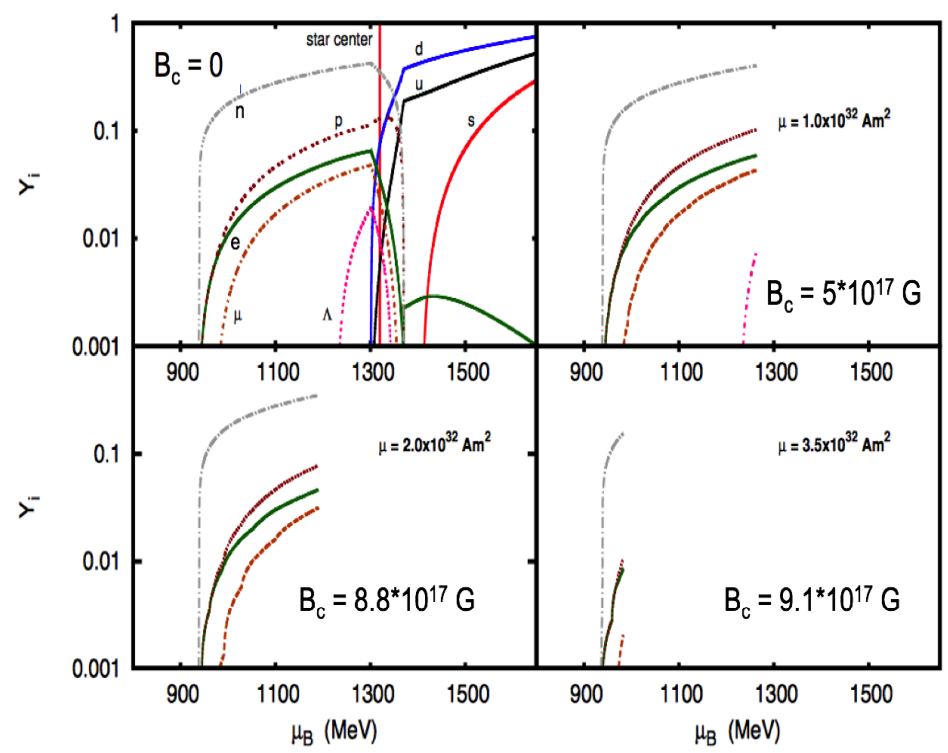

Figure 3: Particle composition of stellar matter as function of baryon chemical potential for different central magnetic fields. The corresponding values for the total stellar magnetic moment $\mu$ is also indicated.

mixed phase have been presented, which show maximum star masses that are in agreement with observation. The approach allows for the calculation of a general equation of state of hot and dense matter that can be used in studies of heavy-ion collisions [10] as well as neutron star mergers [23]. In addition, strong magnetic fields can have a significant effect on the particle content of the star, suppressing exotic degrees of freedom like hyperons and quarks.

\section{Acknowledgements}

SWS acknowledges support from the LOEWE Center HIC for FAIR. Numerical calculations 
were performed at the Center for Scientific Computing of Goethe University, Frankfurt am Main. A. M. was financially supported by the BMBF. Support comes in part from the National Science Foundation under grant PHY-1748621.

\section{References}

[1] B. P. Abbott et al. [LIGO Scientific and Virgo Collaborations], Phys. Rev. Lett. 119 (2017) no.16, 161101

[2] P. Papazoglou et al., Physical Review C 57, 2576 (1998).

[3] P. Papazoglou et al., Physical Review C 59, 411 (1999).

[4] S. Schramm, Physical Review C 66, 064310 (2002).

[5] V. A. Dexheimer and S. Schramm, Physical Review C 81, 045201 (2010).

[6] J. Steinheimer, S. Schramm, and H. Stöcker, Journal of Physics G: Nuclear and Particle Physics 38, 035001 (2011).

[7] K. Fukushima, Physics Letters B 591, 277 (2004).

[8] C. Ratti, M. A. Thaler, and W. Weise, Phys. Rev. D 73, 014019 (2006).

[9] A. Mukherjee, S. Schramm, J. Steinheimer and V. Dexheimer, Astron. Astrophys. 608, A110 (2017)

[10] A. Mukherjee, J. Steinheimer and S. Schramm, Phys. Rev. C 96, no. 2, 025205 (2017)

[11] J. Steinheimer, S. Schramm and H. Stöcker, Phys. Rev. C 84, 045208 (2011)

[12] G. Aarts, C. Allton, D. De Boni, S. Hands, B. Jäger, C. Praki and J. I. Sk ullerud, EPJ Web Conf. 175, 07016 (2018)

[13] G. Aarts, C. Allton, D. De Boni, S. Hands, B. Jäger, C. Praki and J. I. Skullerud, JHEP 1706, 034 (2017)

[14] P. B. Demorest et al., Nature 467, 1081 (2010).

[15] J. Antoniadis et al., Science (New York, N.Y.) 340, 448, 1233232 (2013).

[16] B. Margalit and B. D. Metzger, Astrophys. J. 850, no. 2, L19 (2017).

[17] L. Rezzolla, E. R. Most and L. R. Weih, Astrophys. J. 852, no. 2, L25 (2018)

[18] R. C. Duncan and C. Thompson, Astrophys. J. 392, L9 (1992).

[19] B. Paczynski, Acta Astron. 42, 145 (1992).

[20] A. I. Ibrahim et al., AIP Conf. Proc. 714, 294 (2004).

[21] B. Franzon, V. Dexheimer and S. Schramm, Mon. Not. Roy. Astron. Soc. 456, no. 3, 2937 (2016)

[22] V. Dexheimer, R. Negreiros and S. Schramm, Eur. Phys. J. A 48, 189 (2012)

[23] E. R. Most, L. J. Papenfort, V. Dexheimer, M. Hanauske, S. Schramm, H. Stöcker and L. Rezzolla, arXiv:1807.03684 [astro-ph.HE]. 


\section{DISCUSSION}

GIULIO AURIEMMA QUESTION: Do you have any guess about the role in the EOS of multiquark states?

STEFAN SCHRAMM ANSWER: In general, additional degrees of freedom soften the equation of state, and in turn reduce the maximum mass of neutron stars. Pentaquarks and similar states are most likely too heavy to occur in stars. If there were low-mass bosonic even-number quark states like the often-discussed $\mathrm{H}$ dibaryon, boson condensation effects could soften the EOS significantly generating low-mass stars in conflict with observation. 